\title{
Some suggestions on dealing with measurement error in linkage analyses
}

\author{
Marko Bachl ${ }^{1} \&$ Michael Scharkow ${ }^{2}$ \\ ${ }^{1}$ University of Hohenheim \\ ${ }^{2}$ Zeppelin University
}

Draft, 2018-04-25

Linkage analysis is a sophisticated media effect research design that reconstructs the likely exposure to relevant media messages of individual survey respondents by complementing the survey data with a content analysis. It is an important improvement over survey-only designs: Instead of predicting some outcome of interest by media use and implicitly assuming what kind of media messages the respondents were exposed to, linkage analysis explicitly takes the media messages into account (de Vreese \& Neijens, 2016; Scharkow \& Bachl, 2017; Schuck, Vliegenthart, \& de Vreese, 2016; Shoemaker \& Reese, 1990; Slater, 2016; Valkenburg \& Peter, 2013). The design in its modern form has been pioneered by Miller, Goldenberg, and Erbring (1979) and is today considered a "state-of-the art analysis of the impact of specific news consumption" (Fazekas \& Larsen, 2015, p. 196). Its widespread use, especially in the field of political communication, and its still increasing popularity demonstrate the relevance of the design. The main advantage of a linkage analysis is the use of one or more message exposure variables which combine information about media use and media content. However, both constitutive sources are often measured with error: Survey respondents are not very good at reporting their media use reliably, and coders will often make some errors when classifying the relevant messages.

In this article, we will first give a short overview on the prevalence and consequences of measurement error in both data sources. The arguments are based on a literature review and a simulation study which are published elsewhere in full detail (Scharkow \& Bachl, 2017). We continue with a discussion of possible remedies in measurement and data analysis. Beyond the obvious need to improve the measures themselves, we highlight the importance of serious diagnostics of measurement quality. Such information can then be incorporated in the data analysis using estimation or imputation approaches, which are introduced in the main

Manuscript prepared for publication in 'Measuring Media Use and Exposure: Recent Developments and Challenges', proceedings of the 2016 annual meeting of the DGPuK methods division, edited by Rinaldo Kühne, Teresa Naab, and Christina Peter (http://www.halem-verlag.de/measuring-media-use-and-exposure/). We thank Daniel Oberski for valuable comments and references to additional correction procedures and implementations.

Correspondence concerning this article should be addressed to Marko Bachl, Department of Communication, University of Hohenheim, Stuttgart, Germany. E-mail: marko.bachl@uni-hohenheim.de 
section of this chapter. We conclude by noting that 1) the improvement of measurements and the diagnosis of measurement error in both parts of a linkage analysis must be taken seriously; 2) many tools for correcting measurement error in single parts of a linkage analysis already exist and should be used; 3) methodological research is needed for the development of an integrated analysis workflow which accounts for measurement error and uncertainty in both data sources.

\section{Sources and consequences of measurement error in linkage analysis}

The central part of a linkage analysis is the message exposure variable. ${ }^{1}$ It is a summary of the media messages that were present in the news coverage (according to the content analysis) of the outlets which a respondent has used (according to the survey). The variable approximates each individual's exposure to the relevant media messages, and its effect on the outcome of interest is the media effect estimate. Yet, both components of this variable are subject to measurement error. Studies have shown that media use self-reports have only moderate test-retest reliability (Allen, 1981; Bartels, 1993; Dilliplane, Goldman, \& Mutz, 2013; Lee, Hornik, \& Hennessy, 2008; Scharkow, in prep.) and rather low accuracy (Scharkow, 2016; Wonneberger \& Irazoqui, 2016). With regard to (political) news media use, overreporting is often considered the foremost concern (Price \& Zaller, 1993; Prior, 2009b). The reliability of media message measures in content analyses varies widely depending on the messages of interest. Especially complex and rare message types (e.g., evaluations of the European Union, Desmet, van Spanje, \& de Vreese, 2015; frames, Schuck, Boomgaarden, \& de Vreese, 2013) have been reported to show rather low inter-coder reliability.

Measurement error in the media use self-reports leads to attenuated media effect estimates in simpler studies that predict the outcomes of interest by media use only (Bartels, 1993; Zaller, 2002). The consequences of an unreliable content analysis for media effect studies had not been investigated previously. It is known from probability theory that random measurement error in categorical variables (and therefore in almost all content analysis measures) biases even simple proportion estimates, and that this bias is larger for more unevenly distributed variables (Bachl \& Scharkow, 2017; Schwartz, 1985). We thus assumed that the coding reliability and the prevalence of the relevant messages in the media coverage would influence the ability of a linkage analysis to uncover the true media effect, in addition to the biases introduced by the imperfect survey measures.

We explored the combined influence of measurement error in both constitutive sources with a simulation study (Scharkow \& Bachl, 2017). The reliability of the observed message exposure measure and the relative bias of the observed media effect were assessed for various combinations of measurement error in the content analysis and the survey part of a linkage study. Table 1 highlights the most important findings for reliability levels at the lower bounds which are commonly considered acceptable. Rows 3 and 6 show the well-known attenuating impact of unreliable media use self-reports (Bartels, 1993; Zaller, 2002) when assuming perfect coding. If the true-score reliability of the media use variable decreased to .7 , only $84 \%$ of the true media effect was uncovered. Rows 2 and 5 reveal the consequences of

\footnotetext{
${ }^{1}$ Some linkage studies use more than one message exposure variable which approximate the exposure to different messages or by different types of media outlets. In this article, however, we limit our presentation to the most common case of one message exposure variable. The correction procedures which are discussed in the following section can also be extended to several message exposure variables.
} 
measurement error in the content analysis if respondents were capable of perfectly reporting their media use. Less reliable measurement of the media messages also decreased the reliability of the message exposure measure and attenuated the media effect estimate. The consequences are substantially more severe for rare messages (Row 2) than for relatively common messages (Row 5). If both data sources contained measurement error, the damage accumulated (Rows 1 and 4): For a scenario at the lower bound of conventionally acceptable measures, the reliability of the message exposure variables was less than .6 , and the media effect estimates were reduced to $72 \%$ or $76 \%$, respectively, of their true magnitude. In summary, the simulation showed that realistic quantities of measurement error can lead to substantially downward-biased media effect estimates, because the core feature of the linkage analysis design, the message exposure approximation, becomes rather unreliable. The severity of the bias depends on message frequency (rare messages are more problematic) and the reliability of both media use self-reports and media message measures.

Table 1

Reliability of the observed message exposure measure and relative bias of the observed media effect at typical levels of measurement error and message frequency

\begin{tabular}{rrrrrr}
\hline & \multicolumn{3}{c}{ Input parameters } & \multicolumn{2}{c}{ Outcomes } \\
Row & $p_{\text {Message }}^{\text {true }}$ & $R e l_{\text {Coding }}$ & $R e l_{\text {Media Use }}$ & $R e l_{\text {Message Exposure }}$ & Relative Bias \\
\hline 1 & 0.10 & 0.7 & 0.7 & 0.52 & 0.72 \\
2 & 0.10 & 0.7 & 1.0 & 0.73 & 0.86 \\
3 & 0.10 & 1.0 & 0.7 & 0.71 & 0.84 \\
4 & 0.25 & 0.7 & 0.7 & 0.58 & 0.76 \\
5 & 0.25 & 0.7 & 1.0 & 0.82 & 0.90 \\
6 & 0.25 & 1.0 & 0.7 & 0.71 & 0.84 \\
\hline
\end{tabular}

Notes. The values for the outcomes are means from 1,000 simulation runs. Overreporting is fixed at 0 days (no overreporting), because its consequences were comparatively limited. Input parameters: $p_{\text {Message }}^{\text {true }}$ : True proportion of news items with the relevant message; Rel $_{\text {Coding }}$ : True-score reliability of the coding procedure (proportion of correctly coded news items); $R e l_{\text {Media Use: True-score }}$ reliability of the media use measures; Outcomes: Rel $l_{\text {Message Exposure: True-score }}$ reliability of the message exposure variable; Relative bias: Ratio of observed effect to true effect.

\section{Possible remedies I: Improving measurement}

It is clear that one central recommendation is to develop and to use more reliable measures. In the context of linkage analysis, this concerns both the survey and the content analysis components. Recent work in political and communication science has primarily focused on improving the measurement of media use. For example, the news media use measures in the ANES where discussed extensively by Dilliplane et al. (2013), Goldman, Mutz, and Dilliplane (2013), and Prior (2013). The Communication Methods and Measures special issues edited by Fishbein and Hornik (2008) and de Vreese and Neijens (2016) were also devoted to the challenges of measuring media use. Self-reports of media use in traditional 
surveys may be improved by suitable question format and style (e.g., Andersen, de Vreese, \& Albæk, 2016; Dilliplane et al., 2013) or by providing anchoring statements (e.g., Prior, 2009a). Media diaries as an alternative format of capturing media use with self-reports may also yield more reliable and accurate results (e.g., Ohme, Albaek, \& de Vreese, 2016). The problems of respondents' unreliable recall and subjective biases may be overcome by passive tracking measures of media use (e.g., LaCour \& Vavreck, 2014; Scharkow, 2016; Stark, Magin, \& Jürgens, 2017). Such methods, however, have shortcomings of their own, which are caused foremost by technological limitations of the available tools and high demands on participant cooperation (see the detailed discussion of the method by Stark et al., 2017, pp. 62-72). Overall, while much work needs to be done, the challenges with regard to improving the measurement of media use are taken seriously and already sparked active methodological research.

In contrast, the content analytical measurement of relevant media messages has received comparatively little attention. Shoemaker and Reese (1990) already diagnosed that content analysis lagged behind survey-based research in the systematic development of standardized, comparable measures. The respective paragraphs of published linkage studies' methods sections convey the impression that every research project comes up with another operational definition of the theoretically relevant message features, although many studies in political communication are interested in the effects of similar messages. Re-using codebooks seems to be the exception and is only found within research teams, if at all. This does not even begin to mention the usually undocumented, but presumably diverse instructions, training materials, and test items during coder training and evaluation. Consequently, a first desirable step would be the publication of codebooks as well as materials and data from the reliability tests, so that subsequent projects do not have to re-invent the wheel time and again. This lays the foundation for cumulative development of standard content analytical measures similar to the methodological literature on scale development in survey-based research.

Modern complements or alternatives to traditional manual content analysis also hold many promises for linkage analysis. The classification of messages by computer algorithms is an active field of methodological development and the coding quality seems to be satisfactory for use in applied projects, at least for more simple message categories (Grimmer \& Stewart, 2013). We do not argue that such methods produce inherently more reliable data, but their misclassification error is highly deterministic and can be easily estimated. This property makes them ideal for subsequent error correction procedures, which we propose to use in any case in the following section. Crowd-sourced content analysis, i.e., collecting multiple codings per unit from many coders, is another efficient way to classify many messages (Benoit, Conway, Lauderdale, Laver, \& Mikhaylov, 2016). Again, the data quality of such approaches is not better per se, but they are well suited to derive meaningful diagnostics of the error structure for subsequent correction procedures. In addition, a crowd-sourced content analysis forces the researcher to explicitly state all relevant coding instructions, because there is no interpersonal (and undocumented) coder training step. This should lead to more reproducible instruments, which can be used in future projects. 


\section{Possible remedies II: Correcting for the consequences of measurement error}

Even with improved measurement, some error will almost always remain in the data. Despite of a rich methodological literature on how to mitigate the biases which are introduced to statistical estimates by measurement error (Buonaccorsi, 2010; Fuller, 1987), most linkage analyses in communication research seem to be oblivious to such procedures. In the remainder of this chapter, we provide a concise overview of promising approaches, give references to the relevant literature and software implementations, and describe how some procedures may be integrated in the statistical analysis of a linkage study.

For a first overview, it is helpful to distinguish between two general approaches: estimation and imputation (Table 2). Estimation approaches aim at mitigating the bias in the coefficients and their standard errors within a statistical model. Consequently, they require some knowledge of the error structure and its mechanism in the analytical models. The data set itself remains untouched, only the estimates are corrected. In contrast, imputation approaches generate multiple stand-alone data sets which could have been observed if there was no measurement error. Any statistical model can then be used to analyze the data sets and, finally, the quantities of interest from the data sets are combined. Again, some knowledge of the error structure is necessary to generate the imputed data sets. In addition, merging rules for the quantities of interest are needed.

Table 2

Estimation and imputation approaches to measurement error correction

\begin{tabular}{llll}
\hline Approach & Content Analysis & $\begin{array}{l}\text { Media } \\
\text { Report }\end{array}$ & Use \\
& Melf- & Message Exposure \\
\hline Estimation & MCSIMEX & & $\begin{array}{l}\text { Errors-in-Variables, } \\
\text { (MC)SIMEX }\end{array}$ \\
Imputation & $?$ & $\begin{array}{l}\text { Multiple Overimpu- } \\
\text { tation }\end{array}$ & $\begin{array}{l}\text { Multiple Overimpu- } \\
\text { tation }\end{array}$ \\
\hline
\end{tabular}

Before we continue with a more detailed presentation of some procedures, we note that both estimation and imputation approaches require (approximate) information about the error structure of the variables in question. The ideal scenario is a research design which enables direct measurement error estimation. For (quasi-)continuous measures of media use (e.g., the common "days of a week" question), where the assumption of random normal measurement error seems reasonable enough, the error variance can be estimated with a test-retest model of three-wave panel data (Heise, 1969; D. E. Wiley \& Wiley, 1970). The Heise (1969) model has also been applied to estimate the reliability of dichotomous list responses ("Which of the outlets listed below do you use?") (Dilliplane et al., 2013), a model choice which is, however, not universally agreed upon (Alwin, 2007, Chapter 11). The misclassification error in the content analysis component can be empirically approximated from the data of intercoder reliability tests (Bachl \& Scharkow, 2017). But even without empirical information about the error structure in one or both data sources, sensitivity analyses with different combinations of reasonable best- and worst-case scenarios of measurement error are possible. Plausible values can be taken from general (e.g., Alwin, 2007) 
or topic-specific (e.g., Scharkow (in prep.)) overviews or from similar studies. This would still be more honest than an analysis which is based on the premise that no measurement error had occurred at all.

\section{Estimation: Matrix correction, errors-in-variables-models, and (MC)SIMEX}

Matrix correction. Matrix correction or matrix back-calculation is a straightforward procedure to correct proportion estimates from categorical variables if the misclassification error is known (Fuller, 1987; Kuha \& Skinner, 1997). In another article, we have evaluated the suitability of the procedure for content analytical studies in communication research (Bachl \& Scharkow, 2017). Information from the common intercoder reliability test can be used to approximate the misclassification matrix which characterizes the direction and frequency of correct and incorrect codings given the true values. The coders' classifications can be compared to a preferred standard (e.g., codings by the researchers) or to a majority standard (i.e. the modal category of multiple codings per coding unit). Even in the absence of the raw data from the reliability test, intercoder agreement can be used to approximate the maximum possible accuracy. The simulation study shows that the bias in the proportion estimates can be substantially reduced under many circumstances. The procedure performs especially well if high-quality information about the misclassification process is available from large intercoder tests with many test units, many coders, and, ideally, a preferred standard.

The estimation of the share (or number) of news items with a relevant message is an important intermediate step in a linkage analysis. If, for a simple example, exposure to negative evaluations is assumed to exert an effect, the share of news items with negative evaluations is estimated for each media outlet (see Step 2 in the Appendix of Scharkow \& Bachl, 2017). These proportions can be corrected by matrix back-calculation, before they are used to calculate the individual message exposure scores together with the media use variables. A production-ready software implementation of the matrix correction procedure in Bachl and Scharkow (2017) is not (yet) available, but our supplementary materials document the necessary $R$ code. A simple implementation in $R$ base is

solve(MCM) $\% * \%$ obs_props

where MCM is the misclassification matrix, solve() calculates the inverse of the misclassification matrix, and obs_props is the vector of observed proportions. In Bachl and Scharkow (2017), we used a more elaborated least squares procedure with equality and inequality constraints (Isei() from the limSolve package; Soetaert, Van den Meersche, \& van Oevelen, 2009) to guarantee corrected proportions inside the range between 0 and 1. Similar correction procedures are implemented in Mplus (Asparouhov \& Muthén, 2014) and Latent Gold (Bakk, Tekle, \& Vermunt, 2013), where they are used in the context of latent class analysis. They could also be adapted to correct the estimated proportions from the content analysis part of a linkage study given the observed data and an approximation of the misclassification probabilities.

Errors-in-variables-models. Errors-in-variables-models can and have been used to correct for random normal error in interval-scale variables. For variables with known reliability, it is straightforward to estimate a latent-variable model with a specified amount 
of true and error variance (Bollen, 1989). Bartels (1993) already proposed this method over two decades ago for media effect studies in which the outcomes are predicted by media use self-reports only. The advantages of this approach are that it works in any regressiontype model where media use is a predictor or outcome and it is easy to implement in most statistical software packages. Stata users have the eivreg command readily available (Hardin \& Carroll, 2003; Hardin, Schmiediche, \& Carroll, 2003a). Users of flexible structural equation modelling software can simply fix the error variance of a single indicator latent variable. Again, Stata sem users have the simple reliability() option available.

For example, the eivreg syntax for an errors-in-variables-model where the standardized message exposure variable has a test-retest reliability of .8 (and, consequently, an error variance of .2$)$, is ${ }^{2}$

eivreg post pre exposure, $r$ (exposure .8)

where the $r($ ) part behind the comma defines the reliability of the observed message exposure variable. With the Stata sem command and the reliability() option, the code is

sem (exposure_obs <- exposure_true) (post <- pre exposure_true), reliability (exposure_obs .8)

The respective lavaan (Rosseel, 2012) syntax is

exposure_true $=\sim 1 *$ exposure_obs

exposure_obs .2*exposure_obs

post pre + exposure_true

In both structural equation modeling solutions, exposure_true is defined as a latent variable with a single manifest indicator exposure_obs. The reliability (in Stata sem) or error variance (in lavaan) of the manifest indicator is supplied with a fixed value.

Unfortunately, there is only empirical information about the reliability of the selfreported media use - from the study data itself, if the media use questions are asked in three separate waves, or from similar studies. The reliability of the composite message exposure measure cannot be estimated directly or derived analytically from estimates of the error structures in both constitutive measures. One simple approximation would be to use the simulated results from Scharkow and Bachl (2017) as a look-up table to find plausible values of the message exposure variable's reliability for given levels of misreporting, coding errors, and message prevalence. A more advanced variation of this approach would be to perform a similar simulation with inputs from the empirical study. This approach assumes, however, that the error of the message exposure variable is random normal with a mean of zero - an assumption which is not met in most circumstances, because the misclassification error of the categorical media content measures is not random normal. The combination of matrix correction and an errors-in-variables-model is a viable alternative. After correcting the misclassification error in the media message measure, the remaining error in the message exposure score equals the random normal error in the media use measures and can be accounted for in an errors-in-variables-model.

\footnotetext{
${ }^{2}$ This and all subsequent examples assume for sake of simplicity that the outcome post and its pre-exposure measure pre were measured without error, which is of course an unrealistic scenario. A complete analysis should also take into account measurement error in these variables and any covariates.
} 
(Misclassification) simulation extrapolation ([MC]SIMEX). Simulation extrapolation is a powerful and flexible framework for estimating error-corrected quantities. The basic idea is intuitive: First, a sequence of increasingly biased estimates is obtained by fitting the statistical model to the observed data set and to data sets to which successively more measurement error is added (simulation step). Second, the estimates are extrapolated backwards to the point where no measurement error existed (extrapolation step). The method can deal with continuous variables measured with random normal error (the original SIMEX procedure; Carroll, Küchenhoff, Lombard, \& Stefanski, 1996; Cook \& Stefanski, 1994) and with discrete variables measured with misclassification (the MCSIMEX procedure; Küchenhoff, Lederer, \& Lesaffre, 2007; Küchenhoff, Mwalili, \& Lesaffre, 2006). Similar to matrix correction and errors-in-variables-models, information about the measurement error must be provided. Software implementations are readily available for $R$ (Lederer \& Küchenhoff, 2006) and Stata (only the original SIMEX; Hardin, Schmiediche, \& Carroll, 2003b). The example from above in Stata using the simex command is

local error_var $=.2$

mat error_var_matrix = ('error_var')

simex (post=) (exposure_true: exposure_obs), suuinit(error_var_matrix)

where the first two lines define the error variance of a random normal error and the third line runs the regression with SIMEX correction. Different options for obtaining standard errors and for the extrapolation model are also implemented. The simex() function in $R$ works similarly,

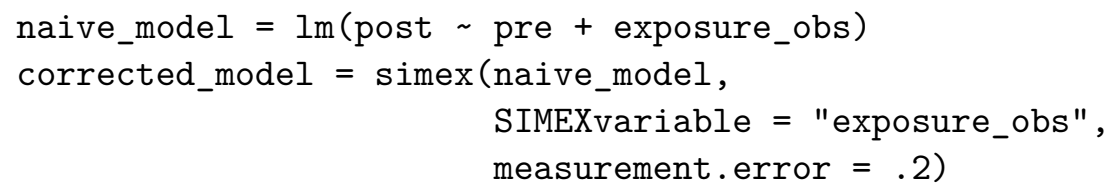

Its flexible nature makes the (MC)SIMEX approach a promising candidate for further linkage-analysis-specific developments. A pipeline which incorporates both the misclassification in the content analysis and the measurement error in the survey seems possible at first glance, but methodological work remains to be done to fully develop and evaluate such an approach.

\section{Multiple overimputation}

Multiple overimputation is an attractive alternative to estimation-based error-correction approaches (Blackwell, Honaker, \& King, 2015a, 2015b). Multiple overimputation extends the idea of multiple imputation of missing values to the mitigation of measurement error. Each observation of a variable is understood to be placed on a continuum which reaches from perfect measurement over increasing grades of measurement error to no measurement at all (i.e., a missing value). The multiple overimputation procedure creates multiple data sets by imputing all values conditional on observation-level priors and all other variables in the data set with an expectation-maximization algorithm. The priors for the mismeasured variables can be estimated within the procedure from gold standard or proxy variables. The more 
likely scenario in an applied linkage analysis is that the test-retest reliability is estimated in a separate reliability model (if three waves of panel data are available) or else taken from the reliability assessment of a similar measure.

Multiple overimputation has some advantages over estimation-based methods. The procedure is, in comparison, quite robust to violations of the random normal error assumption and less dependent on the analytical and correction models (Blackwell et al., 2015a). It might also be easier to use for some researchers, because the error correction step is somewhat separated from the analysis step. After the creation of multiple overimputed data sets, the researcher is free to perform any data transformations and to use almost any familiar statistical model just like with a "normal" data set. Only in the end, the estimates from the multiple overimputed data sets are again combined following Rubin's rule, similar to simple multiple imputation. However, it is important to note that all model variables have to be included in the overimputation procedure. Consequently, the researchers must be able to identify all relevant variables and (approximate) information on their measurement error. As a plus, the procedure not only takes measurement error into account, but also deals with missing values.

Multiple overimputation and the combination of estimates from multiple data sets is implemented the $R$ package Amelia (Honaker, King, \& Blackwell, 2011). Overimputed data sets from Amelia are also compatible with the Stata program Clarify (King, Tomz, \& Wittenberg, 2000), which can be used to analyze the data sets and to combine the estimates. The overimputation step for the media use variables in a survey data set, $\mathrm{m} 1, \mathrm{~m} 2, \ldots, \mathrm{m} 10$, and an assumed reliability of .8 for each variable is carried out in Amelia with the syntax

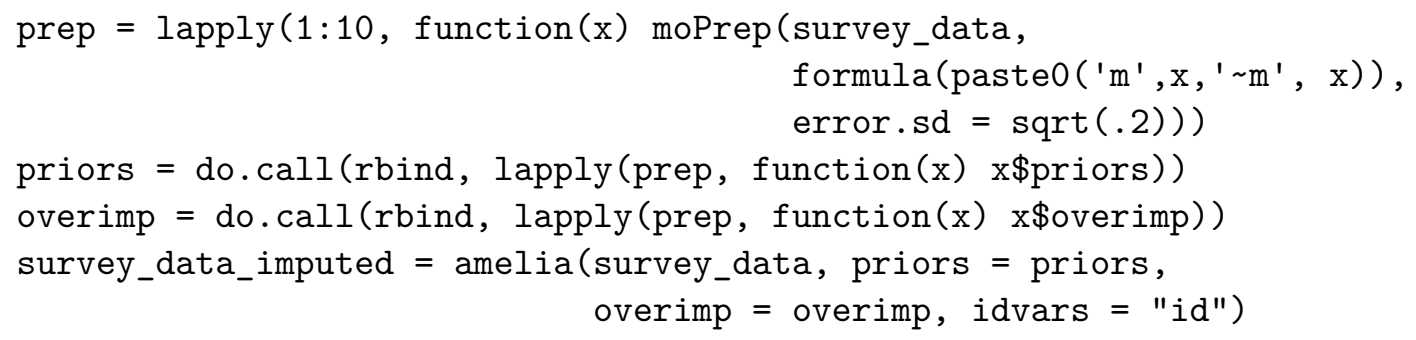

where the first three lines define the error variance (priors) and the variables to be overimputed (overimp) and the fourth line creates a list object with the overimputed data sets.

The major shortcoming of multiple overimputation for our purpose is its inability to correct for misclassification error in nominal variables. Blackwell et al. (2015a) only showed that the method works well for ordinal categorical variables. Consequently, the procedure cannot be used for most of the common media message measures. It can still be a useful approach when combined with matrix correction of the content analytical estimates. One could merge the matrix-corrected media message estimates with the overimputed data sets, compute the message exposure scores, conduct the analysis, and finally combine the estimates from the data sets. 


\section{Where to go from here?}

\section{Recommendations for the practice of linkage analysis}

As we have illustrated in the previous sections, a variety of relatively easy to use methods for correcting measurement errors have been developed in recent years. These provide solutions for categorical and continuous variables with random or systematic error structures. Ideally, however, we should try to reduce measurement error as early as possible instead of dealing with it after the fact. Our first recommendation is therefore to take measurement seriously, to test, re-test and validate measures in different samples and contexts. In survey research as well as in psychometrics and educational testing, there is a rich tradition of measurement theory, survey development and response modeling. Lots can be gained from applying this knowledge to the measurement of media use, but also in content analysis. Moreover, since linkage analyses involve many steps for which a variety of options are available, we argue that a standardization of procedures would greatly enhance the replicability and generalizability of such studies. Such a standardization is our second recommendation.

A more specific advice we can offer is to include reliability and validity checks into the study design of any linkage analysis: Collecting panel data with at least three measurement occasions enables researchers to assess the test-retest reliability of measures directly, and using multiple indicators instead of single items will generally increase the reliability and validity of these measures. For the content analysis, we recommend conducting several reliability tests before and during the coding, and to conduct reliability tests as large as possible, both in terms of coding units (especially for rare categories) and coders. As we have shown elsewhere (Bachl \& Scharkow, 2017), error correction works best when reliable information on the misclassification process is available. This can also be achieved by the collection of true "gold standard" data, i.e. from expert coders or the primary researchers.

Concerning the procedures introduced in this paper, we currently recommend the following strategy: Correct content analysis estimates using matrix correction (Bachl \& Scharkow, 2017) or MCSIMEX, because misclassification of categorical variables does not involve a random normal error. The corrected estimates can easily be saved and used in the linkage step. If you can, use multiple overimputation on the media use self-reports using estimates (e.g. from 3 -wave panel data) or reasonable assumptions, and use the $\sim 10$ imputed data sets for following linkage and analysis steps. Alternatively, it is also possible to combine the corrected content analysis estimates with the uncorrected self-reports, and then use errors-in-variables regression, SIMEX or multiple overimputation with the resulting exposure scores, since the remaining error will be similar to the error in the basic media use variables. Furthermore, remember that the outcome variables and any covariates are rarely error-free, which can be accounted for either before (via multiple overimputation) or during estimation of the final model. We also recommend to conduct robustness checks using different best- and worst-case assumptions about the reliability of the measures in the study. This can also help in allocating resources, e.g. for better coder training, more exposure measures, larger media samples etc. in future studies.

\section{Future methodological research}

In order to be widely adopted, correction procedures need to be well-understood and tested, but also easy to use for the applied researcher. While the procedures outlined above are not 
difficult to apply, most communication scholars would probably welcome a unified approach that provides a single set of corrected estimates with standard errors and/or confidence intervals for the effects of interest. The current multi-step approaches we have just suggested cannot provide this, not because multiple steps are required, but because some information is lost due to aggregation and linkage. In order to obtain correct estimates and standard errors, multiple sources of uncertainty (e.g. about the misclassification error in the content analysis, or the reliability of the media use measures) and sampling variability (from the content analysis and the survey sample) need to be accounted for in the final model. For example, if the reliability test for coding was small, the resulting agreement measures will vary considerably, and so will the corrected estimates using MCSIMEX or matrix correction. However, since the linkage step relies only on the point estimates, such uncertainty will not impact the estimates of the linkage analysis: The results will not differ between a study employing two coders and a handful of test documents and a large-scale analysis using crowdcoding and a large test set. The same is true for the correction of the media use or exposure scores. Since only point estimates of the test-retest reliability are used, their precision does not matter. This is obviously a problem, and will likely lead to standard errors that are too small, increasing the risk of type I errors.

The only solution to this problem is to account for uncertainty and variability throughout the correction and linkage steps. For errors-in-variables models, this problem has been discussed by Oberski and Satorra (2013). For misclassification models, we suggest looking into Bayesian models based on Dawid and Skene (1979) and Passonneau and Carpenter (2014). Ultimately, a full Bayesian model using all the data to simultaneously estimate and correct for measurement error seems the most promising approach to the problem, although it might be a while until an out-of-the-box solution is available to communication scholars. As always, further research is needed. 


\section{References}

Allen, R. L. (1981). The reliability and stability of television exposure. Communication Research, 8(2), 233-256. doi:10.1177/009365028100800205

Alwin, D. F. (2007). Margins of error. A study of reliability in survey measurement. Hoboken: Wiley.

Andersen, K., de Vreese, C. H., \& Albæk, E. (2016). Measuring media diet in a high-choice environment - Testing the list-frequency technique. Communication Methods and Measures, 10(2-3), 81-98. doi:10.1080/19312458.2016.1150973

Asparouhov, T., \& Muthén, B. (2014). Auxiliary variables in mixture modeling: Three-step approaches using Mplus. Structural Equation Modeling: A Multidisciplinary Journal, 21(3), 329-341. doi:10.1080/10705511.2014.915181

Bachl, M., \& Scharkow, M. (2017). Correcting measurement error in content analysis. Communication Methods and Measures, 11(2), 87-104. doi:10.1080/19312458.2017.1305103

Bakk, Z., Tekle, F. B., \& Vermunt, J. K. (2013). Estimating the association between latent class membership and external variables using bias-adjusted three-step approaches. Sociological Methodology, 43(1), 272-311. doi:10.1177/0081175012470644

Bartels, L. M. (1993). Messages received: The political impact of media exposure. American Political Science Review, 87(2), 267-285. doi:10.2307/2939040

Benoit, K. R., Conway, D., Lauderdale, B. E., Laver, M., \& Mikhaylov, S. (2016). Crowdsourced text analysis: Reproducible and agile production of political data. American Political Science Review, 110(2), 278-295. doi:10.1017/S0003055416000058

Blackwell, M., Honaker, J., \& King, G. (2015a). A unified approach to measurement error and missing data: Details and extensions. Sociological Methods $\&$ Research. doi:10.1177/0049124115589052

Blackwell, M., Honaker, J., \& King, G. (2015b). A unified approach to measurement error and missing data: Overview and applications. Sociological Methods $\&$ Research. doi: $10.1177 / 0049124115585360$

Bollen, K. A. (1989). Structural equations with latent variables. New York: Wiley.

Buonaccorsi, J. P. (2010). Measurement error: Models, methods, and applications. Boca Raton: CRC Press.

Carroll, R. J., Küchenhoff, H., Lombard, F., \& Stefanski, L. A. (1996). Asymptotics for the SIMEX estimator in nonlinear measurement error models. Journal of the American Statistical Association, 91(433), 242-250. doi:10.1080/01621459.1996.10476682

Cook, J. R., \& Stefanski, L. A. (1994). Simulation-extrapolation estimation in parametric measurement error models. Journal of the American Statistical Association, 89(428), 1314-1328. doi:10.1080/01621459.1994.10476871

Dawid, A. P., \& Skene, A. M. (1979). Maximum likelihood estimation of observer error-rates using the EM algorithm. Journal of the Royal Statistical Society. Series C (Applied Statistics), 28(1), 20-28. doi:10.2307/2346806

de Vreese, C. H., \& Neijens, P. (2016). Measuring media exposure in a changing communications environment. Communication Methods and Measures, 10(2-3), 69-80. doi:10.1080/19312458.2016.1150441

Desmet, P., van Spanje, J., \& de Vreese, C. H. (2015). Discussing the democratic deficit: 
Effects of media and interpersonal communication on satisfaction with democracy in the European Union. International Journal of Communication, 9, 3177-3198. doi:1932-8036/20150005

Dilliplane, S., Goldman, S. K., \& Mutz, D. C. (2013). Televised exposure to politics: New measures for a fragmented media environment. American Journal of Political Science, 57(1), 236-248. doi:10.1111/j.1540-5907.2012.00600.x

Fazekas, Z., \& Larsen, E. G. (2015). Media content and political behavior in observational research: A critical assessment. British Journal of Political Science, 46(1), 195-204. doi:10.1017/s000712341500006x

Fishbein, M., \& Hornik, R. (2008). Measuring media exposure: An introduction to the special issue. Communication Methods and Measures, 2(1-2), 1-5. doi:10.1080/19312450802095943

Fuller, W. A. (1987). Measurement error models. New York: Wiley.

Goldman, S. K., Mutz, D. C., \& Dilliplane, S. (2013). All virtue is relative: A response to Prior. Political Communication, 30 (4), 635-653. doi:10.1080/10584609.2013.819540

Grimmer, J., \& Stewart, B. M. (2013). Text as data: The promise and pitfalls of automatic content analysis methods for political texts. Political Analysis, 21(3), 267-297. doi:10.1093/pan/mps028

Hardin, J. W., \& Carroll, R. J. (2003). Measurement error, GLMs, and notational conventions. Stata Journal, 3(4), 329-341. Retrieved from http://www.stata-journal.com/article. html?article $=$ st0047

Hardin, J. W., Schmiediche, H., \& Carroll, R. J. (2003a). The regression-calibration method for fitting generalized linear models with additive measurement error. Stata Journal, 3(4), 361-372. Retrieved from http://www.stata-journal.com/article.html?article= st0050

Hardin, J. W., Schmiediche, H., \& Carroll, R. J. (2003b). The simulation extrapolation method for fitting generalized linear models with additive measurement error. Stata Journal, 3(4), 373-385. Retrieved from http://www.stata-journal.com/article.html? article $=$ st0051

Heise, D. R. (1969). Separating reliability and stability in test-retest correlation. American Sociological Review, 93-101.

Honaker, J., King, G., \& Blackwell, M. (2011). Amelia II: A program for missing data. Journal of Statistical Software, 45(7), 47. doi:10.18637/jss.v045.i07

King, G., Tomz, M., \& Wittenberg, J. (2000). Making the most of statistical analyses: Improving interpretation and presentation. American Journal of Political Science, 44 (2), 347-361. doi:10.2307/2669316

Kuha, J., \& Skinner, C. (1997). Categorical data analysis and misclassification. In L. E. Lyberg, P. Biemer, M. Collins, E. D. De Leeuw, C. Dippo, N. Schwarz, \& D. Trewin (Eds.), Survey measurement and process quality (pp. 633-670). New York: Wiley.

Küchenhoff, H., Lederer, W., \& Lesaffre, E. (2007). Asymptotic variance estimation for the misclassification SIMEX. Computational Statistics \& Data Analysis, 51(12), 6197-6211. doi:10.1016/j.csda.2006.12.045

Küchenhoff, H., Mwalili, S. M., \& Lesaffre, E. (2006). A general method for dealing with misclassification in regression: The misclassification SIMEX. Biometrics, 62(1), 
85-96. doi:10.1111/j.1541-0420.2005.00396.x

LaCour, M. J., \& Vavreck, L. (2014). Improving media measurement: Evidence from the field. Political Communication, 31 (3), 408-420. doi:10.1080/10584609.2014.921258

Lederer, W., \& Küchenhoff, H. (2006). A short introduction to the SIMEX and MCSIMEX. $R$ News, 6(4), 26-31.

Lee, C.-J., Hornik, R., \& Hennessy, M. (2008). The reliability and stability of general media exposure measures. Communication Methods and Measures, 2(1-2), 6-22. doi:10.1080/19312450802063024

Miller, A. H., Goldenberg, E. N., \& Erbring, L. (1979). Type-set politics: Impact of newspapers on public confidence. American Political Science Review, 73(1), 67-84. doi: $10.2307 / 1954731$

Oberski, D. L., \& Satorra, A. (2013). Measurement error models with uncertainty about the error variance. Structural Equation Modeling: A Multidisciplinary Journal, 20(3), 409-428. doi:10.1080/10705511.2013.797820

Ohme, J., Albaek, E., \& de Vreese, C. H. (2016). Exposure research going mobile: A smartphone-based measurement of media exposure to political information in a convergent media environment. Communication Methods and Measures, 10(2-3), 135-148. doi:10.1080/19312458.2016.1150972

Passonneau, R. J., \& Carpenter, B. (2014). The benefits of a model of annotation. Transactions of the Association for Computational Linguistics, 2, 311-326. Retrieved from https://www.transacl.org/ojs/index.php/tacl/article/view/389

Price, V., \& Zaller, J. (1993). Who gets the news? Alternative measures of news reception and their implications for research. Public Opinion Quarterly, 57(2), 133-164. doi:10.1086/269363

Prior, M. (2009a). Improving media effects research through better measurement of news exposure. The Journal of Politics, 71(3), 893-908. doi:10.1017/S0022381609090781

Prior, M. (2009b). The immensely inflated news audience: Assessing bias in self-reported news exposure. Public Opinion Quarterly, 73(1), 130-143. doi:10.1093/poq/nfp002

Prior, M. (2013). The challenge of measuring media exposure: Reply to Dilliplane, Goldman, and Mutz. Political Communication, 30(4), 620-634. doi:10.1080/10584609.2013.819539

Rosseel, Y. (2012). lavaan: An R package for structural equation modeling. Journal of Statistical Software, 48(2), 1-36. doi:10.18637/jss.v048.i02

Scharkow, M. (2016). The accuracy of self-reported Internet use - A validation study using client log data. Communication Methods and Measures, 10(1), 13-27. doi:10.1080/19312458.2015.1118446

Scharkow, M. (in prep.). The reliability and temporal stability of self-reported media use - A meta-analysis.

Scharkow, M., \& Bachl, M. (2017). How measurement error in content analysis and self-reported media use leads to minimal media effect findings in linkage analyses: A simulation study. Political Communication, 34(3), 323-343. doi:10.1080/10584609.2016.1235640

Schuck, A. R. T., Boomgaarden, H. G., \& de Vreese, C. H. (2013). Cynics all around? The impact of election news on political cynicism in comparative perspective. Journal of 
Communication, 63(2), 287-311. doi:10.1111/jcom.12023

Schuck, A. R. T., Vliegenthart, R., \& de Vreese, C. H. (2016). Matching theory and data: Why combining media content with survey data matters. British Journal of Political Science, 46(1), 205-2013. doi:10.1017/S0007123415000228

Schwartz, J. (1985). The neglected problem of measurement error in categorical data. Sociological Methods \& Research, 13(4), 435-466. doi:10.1177/0049124185013004001

Shoemaker, P. J., \& Reese, S. D. (1990). Exposure to what? Integrating media content and effects studies. Journalism \& Mass Communication Quarterly, 67(4), 649-652. doi:10.1177/107769909006700418

Slater, M. D. (2016). Combining content analysis and assessment of exposure through self-report, spatial, or temporal variation in media effects research. Communication Methods and Measures, 10(2-3), 173-175. doi:10.1080/19312458.2016.1150969

Soetaert, K., Van den Meersche, K., \& van Oevelen, D. (2009). limSolve: Solving linear inverse models. Retrieved from https://cran.r-project.org/package=limSolve

Stark, B., Magin, M., \& Jürgens, P. (2017). Ganz meine Meinung? Informationsintermediäre und Meinungsbildung - Eine Mehrmethodenstudie am Beispiel von Facebook. Düsseldorf: Landesanstalt für Medien Nordrhein-Westfalen. Retrieved from http: //lfmpublikationen.lfm-nrw.de/index.php?view=product_detail\&product_id=492

Valkenburg, P. M., \& Peter, J. (2013). Five challenges for the future of media-effects research. International Journal of Communication, 7, 197-215. doi:1932-8036/2013FEA0002

Wiley, D. E., \& Wiley, J. A. (1970). The estimation of measurement error in panel data. American Sociological Review, 35(1), 112-117. doi:10.2307/2093858

Wonneberger, A., \& Irazoqui, M. (2016). Explaining response errors of self-reported frequency and duration of TV exposure through individual and contextual factors. Journalism \& Mass Communication Quarterly, 94(1), 259-281. doi:10.1177/1077699016629372

Zaller, J. (2002). The statistical power of election studies to detect media exposure effects in political campaigns. Electoral Studies, 21 (2), 297-329. doi:10.1016/S02613794(01)00023-3 